\title{
Heuristic Based Adaptive Step Size CLMS ALGORITHMS FOR SMART ANTENNAS
}

\author{
Y Rama Krishna ${ }^{1}$, PV Subbaiah ${ }^{2}$ and B Prabhakara Rao ${ }^{3}$ \\ ${ }^{1}$ PVP Siddhartha Institute of Technology, Vijayawada, India \\ ramakrishna2712@hotmail.com \\ ${ }^{2}$ Amrita Sai Institute of Science \& Technology, Vijayawada, India \\ pvs_ece2000@yahoo.co.in \\ ${ }^{3}$ JNT University Kakinada, Kakinada, India \\ drbprerediffmail.com
}

\begin{abstract}
A smart antenna system combines multiple antenna elements with a signal processing capability to optimize its radiation and/or reception pattern automatically in response to the signal environment through complex weight selection. The weight selection process to get suitable Array factor with low Half Power Beam Width (HPBW) and Side Lobe Level (SLL) is a complex method. The aim of this task is to design a new approach for smart antennas to minimize the noise and interference effects from external sources with least number of iterations. This paper presents Heuristics based adaptive step size Complex Least Mean Square (CLMS) model for Smart Antennas to speedup convergence. In this process Benveniste and Mathews algorithms are used as heuristics with CLMS and the improvement of performance of Smart Antenna System in terms of convergence rate and array factor are discussed and compared with the performance of CLMS and Augmented CLMS (ACLMS) algorithms.
\end{abstract}

\section{KEYWORDS}

Adaptive step size, CLMS, ACLMS, Benveniste Algorithm, Mathews algorithm.

\section{INTRODUCTION}

Wireless cellular networks are fast growing technology in the current world and this trend is likely to continue for several years [1]. The advancements in radio technology enable novel and improved services in the cellular systems. Current wireless services include transmission of voice, fax, and multimedia applications and so on. Multimedia services like video-on demand and internet access needs more band width. Wireless networks must provide these services in a wide range of environments, spanning dense urban, suburban, and rural areas.

Smart Antennas (SA) consist of an array of antenna elements with signal processing capability that optimizes the radiation and reception of a desired signal dynamically. SAs can place nulls in the direction of interferers via adaptive updating of weights linked to each antenna element. SAs thus cancel out most of the co-channel interference, resulting the better quality of reception and lowered dropped calls [2]. SAs can also track the user within a cell via direction of arrival algorithms.

David C. Wyld (Eds) : ICCSEA, SPPR, CSIA, WimoA - 2013

pp. 313-320, 2013. @ CS \& IT-CSCP 2013

DOI : $10.5121 /$ csit.2013.3532 
The Smart antennas perform spatial filtering, which makes it possible to receive energy from a particular direction, while simultaneously blocking it from another direction. This property makes smart antennas as an effective tool in detecting and locating radiation from other sources. That means, the design and development of the efficient models to this task for real time optimization is a current problem. The control unit of the Smart Antenna is normally realized with a Digital Signal Processing (DSP) unit. Based on certain inputs, the DSP algorithm controls radiation parameters of the antenna to optimize communication link. Figure 1 shows the basic model of Smart Antenna systems.

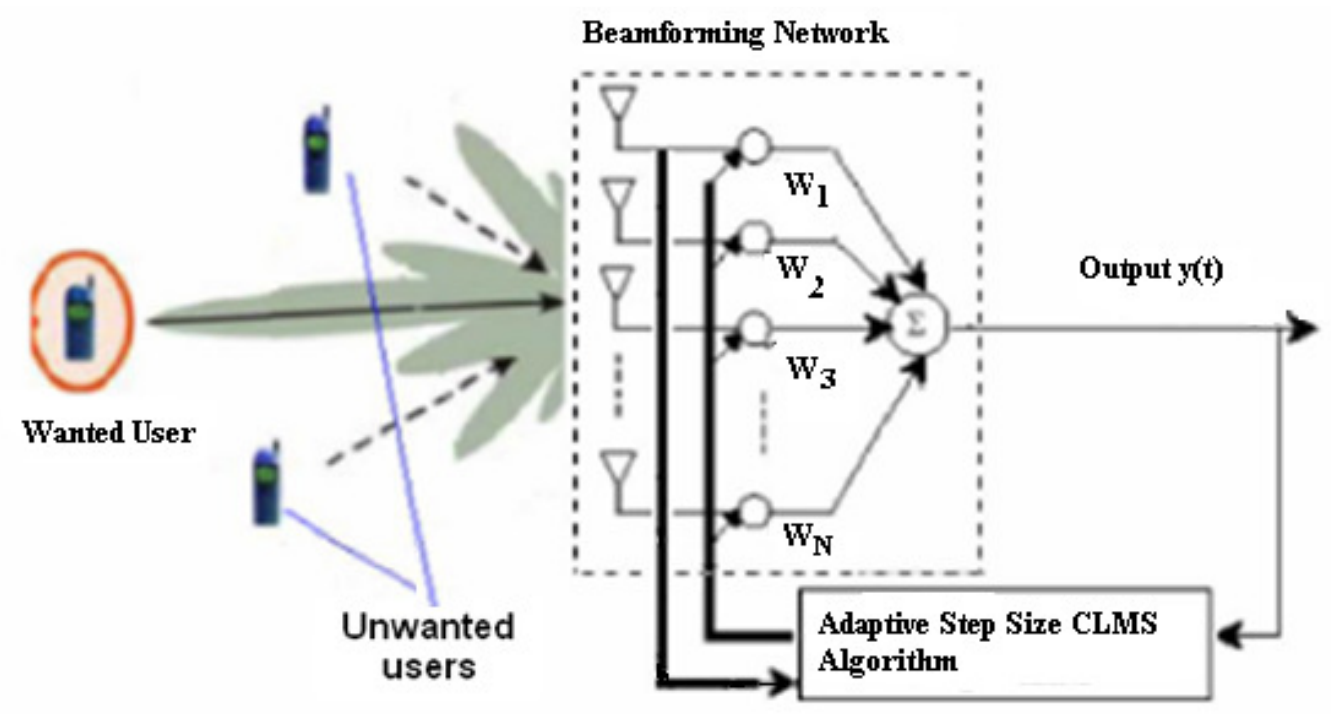

Figure 1. Basic smart antenna system

\section{COMPLEX NeURAL AlgorithMS}

A Complex Valued Neural Network [5, 7] is an artificial neural network, consists of complex valued input signals, weights, threshold values and/or signal functions. Such kind of models must be needed for solving the problems in the field of signal processing. In the signal processing, signals are complex valued and processing of such signals requires the implementation of new complex valued neural processing models. One of the most important characteristics of the complex valued neural models is their ability to process linear complex signals of the smart antennas. In smart antennas signals from different sources or interferers are to be processed before orienting the main beam direction of the antenna array. In this context identifying the angle of arrival of the desired signal is very important. More over the HPBW and SLL of the array radiation pattern must be as small as possible to avoid the interference.

In the work [7], neural models like LMS and ACLMS are selected for signal processing in smart antennas. The LMS algorithm is one of the most common approaches to train linear filters. Despite its robustness, this algorithm is relatively slow at converging to the optimal least squares solution. Where as ACLMS is an augmented version of CLMS and from the results it is observed that, ACLMS is outperforming the CLMS particularly in noisy environments. In noisy environments the value of $\mu$ has to be chosen very small where as in case of noiseless environment, a high value for $\mu$ may be chosen. The importance for selecting $\mu$ value comes from the convergence rate of the array output towards the desired signal. When the $\mu$ value is small and fixed, the algorithm takes more number of iterations and it takes much time to converge. Hence a tool is necessary to speedup the convergence of linear adaptive filters. Adaptive stepsize or 
variable stepsize algorithms can be used as good tool for this purpose [3]. Generally the idea behind adaptive stepsize is to have large stepsizes when the estimated errors are large at the early stages of adaptation, and smaller stepsizes when approaching steady-state convergence and misadjustment [4].

To this end, Benveniste et al. propose and analyse an adaptive stepsize algorithm based on the gradient of the instantaneous squared error with respect to the stepsize. Benveniste's algorithm, in fact, performs time-varying low pass filtering of the noisy instantaneous gradients in the update of the stepsize. This algorithm was derived rigorously without making the usual independence assumptions, which results in better performance but increased computational complexity as compared to standard LMS. Attempts to reduce the computational complexity of this algorithm include the Mathews and Xie algorithm. In Mathews and Xie algorithm, only raw instantaneous gradients are used, which makes this algorithm sensitive to initial conditions and noise. One advantage of Mathews and Xie algorithm over Benveniste's algorithm is their relative simplicity, at the cost of possible performance degradation [9].

\subsection{Benveniste Adaptive Step-size Algorithm}

Consider a stochastic gradient weight update for a nonlinear adaptive finite impulse response filter, given by

$$
w(k+1)=w(k)+\mu(k) e(k) \emptyset^{j *}\left(\left(x^{T}(k) w(k) x^{*}(k)\right)\right.
$$

The parameter $\mu$ is a stepsize (possibly adaptive) and is critical to converge.

To cater for the unknown dynamics of the inputs and their possible nonstationary nature, it is proposed to make the step size $\mu$ gradient adaptive, as

$$
\mu(k)=\mu(k-1)-\rho \nabla_{\mu} J(k)_{\mu=\mu(k-1)}
$$

Where parameter $\rho$ is stepsize. Based on the standard cost function $J(k)=\frac{1}{2}|\mathrm{e}(\mathrm{k})|^{2}=$ $\frac{1}{2} e(k) \mathrm{e}^{*}(\mathrm{k})$, the gradient $\nabla_{\mu} J(k)$ is calculated from

$$
\nabla_{\mu} J(k)=\frac{1}{2}\left[\mathrm{e}(\mathrm{k}) \frac{\partial \mathrm{e}^{*}(\mathrm{k})}{\partial \mu(k-1)}+\mathrm{e}^{*}(\mathrm{k}) \frac{\partial \mathrm{e}(\mathrm{k})}{\partial \mu(k-1)}\right]
$$

The main issue in the derivation of the gradient adaptive step-size algorithms is the calculation of the partial derivatives $\partial \mathrm{e}^{*}(\mathrm{k}) / \partial \mu(k-1)$ and $\partial e(\mathrm{k}) / \partial \mu(k-1)$ from the equation 2 . For instance, to calculate the term $\partial \mathrm{e}^{*}(\mathrm{k}) / \partial \mu(k-1)$, we need to evaluate

$$
\frac{\partial \mathrm{e}^{*}(\mathrm{k})}{\partial \mu(k-1)}=\frac{\partial \mathrm{e}_{\mathrm{r}}(\mathrm{k})}{\partial \mu(k-1)}-\mathrm{j} \frac{\partial \mathrm{e}_{\mathrm{i}}(\mathrm{k})}{\partial \mu(k-1)}
$$

For fully complex analytic nonlinear activation function, by using the Cauchy - Riemann equations, this yields

$$
\frac{\partial \mathrm{e}^{*}(\mathrm{k})}{\partial \mu(k-1)}=-\mathrm{x}^{\mathrm{H}}(\mathrm{k}) \emptyset^{\prime *}(\mathrm{k}) \frac{\partial \mathbf{w}^{*}(\mathrm{k})}{\partial \mu(k-1)}
$$

By a similar calculation, the second gradient term in equation $3, \partial e(\mathrm{k}) / \partial \mu(k-1)$ becomes 


$$
\frac{\partial \mathrm{e}(\mathrm{k})}{\partial \mu(k-1)}=-\mathbf{x}^{\mathrm{T}}(\mathrm{k}) \emptyset^{\prime}(\mathrm{k}) \frac{\partial \mathbf{w}(\mathrm{k})}{\partial \mu(k-1)}
$$

For simplicity,

$$
\psi(\mathrm{k})=\frac{\partial \mathrm{w}(\mathrm{k})}{\partial \mu(k-1)} \cong \frac{\partial \mathrm{w}(\mathrm{k})}{\partial \mu(k)}
$$

Based on weight update (equation 1), equation 8 can be written as

$$
\begin{aligned}
& \psi(\mathrm{k})=\psi(\mathrm{k}-1)+\frac{\partial \mu(k-1)}{\partial \mu(k-1)} e(k-1) \emptyset^{j *}(k-1) x^{*}(k-1)+\mu(k-1) \frac{\partial \mathrm{e}(k-1)}{\partial \mu(k-1)} \emptyset^{j *}(k- \\
& 1) x^{*}(k-1)+\mu(k-1) \mathrm{e}(k-1) \frac{\partial \emptyset^{j *}(k-1)}{\partial \mu(k-1)} x^{*}(k-1)
\end{aligned}
$$

The last term may be neglected, to give

$\psi(\mathrm{k})=\left[\mathrm{I}-\mu(k-1) \mid\left(\left.\emptyset^{\prime}(k-1)\right|^{2} x^{*}(k-1) x^{T}(k-1)\right] \psi(\mathrm{k}-1)+e(k-1) \emptyset^{j *}(k-\right.$ 1) $x^{*}(k-1)$

Based on stepsize adaptation in equation no. 2, The Benveniste's Adaptive step size algorithm becomes

$$
\mu(k)=\mu(k-1)+\frac{\rho}{2}\left[e(k) \emptyset^{\prime *}(\mathrm{k}) x^{H}(k) \psi^{*}(k)+e^{*}(k) \emptyset^{\prime}(\mathrm{k}) x^{T}(k) \psi(k)\right]
$$

\subsection{Farhang-Ang Adaptive Step-size Algorithm}

To simplify Benveniste's adaptive stepsize algorithm update, Farhang and And replaced the time varying term in the square brackets in equation 9 by a constant $0<\alpha<1$. With this simplification, the sensitivity term $\psi(k)$ becomes

$$
\psi(k)=\alpha \psi(k-1)+e(k-1) \emptyset^{\prime *}(k-1) x^{*}(k-1)
$$

This way, the noisy instantaneous gradients $e(k-1) \emptyset^{*}(k-1) x^{*}(k-1)$ are filtered with a low pass filter with a fixed coefficient $\alpha[8]$.

\subsection{Mathews Adaptive Step-size Algorithm}

By setting $\alpha=0$, calculation of the sensitivity term in equation 11 , can be further simplified, to obtain the Mathews adaptive step size algorithm [6], for which

$$
\psi(k)=e(k-1) \emptyset^{\prime *}(k-1) x^{*}(k-1)
$$

is based only on the noisy instantaneous estimates of the gradient from equation 6.

\section{PerformanCe Analysis}

The simulations are carried out with an input signal $x_{s}(k)=\cos (2 w t)$ at a frequency of $1 \mathrm{kHz}$ along with a random noise. When the values of $N$ (number of array elements) and $\mu$ (step size parameter) are varied, one can generate main lobe in required direction with low SLL and HPBW. When the $\mu$ value is increased it is observed that, HPBW and SLL, both are reducing [7]. This scenario is observed for both stationary and non-stationary environments with several core 
algorithms such as RLS, DMI, LMS, CLMS, ACLMS etc. However these algorithms performance is more or less equal in the convergence rate. In order to increase the convergence rate better, some heuristics such as Benveniste and Mathews are considered with an additional parameter "Step size of adaptive amplitude $(\rho)$ ". In this paper, these two algorithms are implemented on Smart Antenna system, giving fast convergence for the output signal than the core models. These two heuristics are giving almost same performance in case of HPBW and SLL where as in case of convergence rate, these are outperforming the core algorithms. The results are presented in figures 2-6.

The results obtained with Benveniste and Mathews algorithms and their comparison with CLMS and ACLMS algorithms in noisy environment, is presented in table 1. From various observations, the stepsize parameter $\mu$ value is fixed at 0.002 and the initial value of adaptive step size amplitude $(\rho)$ is fixed at 0.001 . The reduction of $\rho$ value in Benveniste algorithm results in fast convergence of array output where as the reduction of $\rho$ value in Mathews algorithm does not improve the convergence rate as to the extent of Benveniste algorithm. Hence it may stated that Mathews algorithm is less complex when compared with Benveniste algorithm however at very low values of $\rho$, it is not able to pick up the speed of Benveniste algorithm. Hence Benveniste variable step size model can be adapted for smart antenna system along with neural algorithms like ACLMS and CLMS to speed up the convergence of the array output. These models are also tested for noiseless environment where the same trend of results is observed for both of these heuristic models, and it is shown in the figure 7.

Table1. Performance comparison of Benveniste and Mathews algorithms with CLMS \& ACLMS Algorithms in noisy environment

\begin{tabular}{|c|c|c|c|c|c|}
\hline $\begin{array}{c}\text { Heuristic } \\
\text { function }\end{array}$ & $\mathrm{N}$ & $\mu$ & $\rho$ & HPBW & SLL \\
\hline Benveniste & 8 & 0.002 & 0.000001 & 6.4 & 0.2418 \\
\hline Mathews & 8 & 0.002 & 0.000001 & 6.4 & 0.2337 \\
\hline CLMS & 8 & 0.002 & -- & 6.8 & 0.2106 \\
\hline ACLMS & 8 & 0.002 & -- & 7.0 & 0.1622 \\
\hline
\end{tabular}

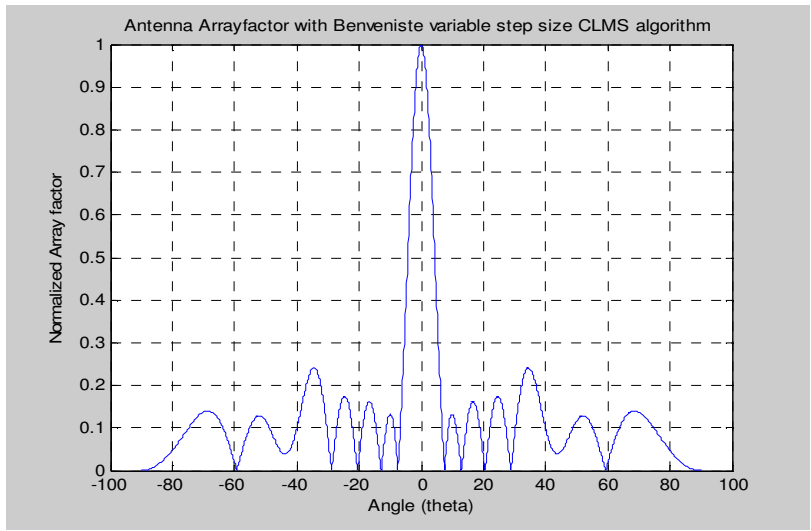

Figure 2. Antenna Array factor with Benveniste variable step size CLMS algorithm 


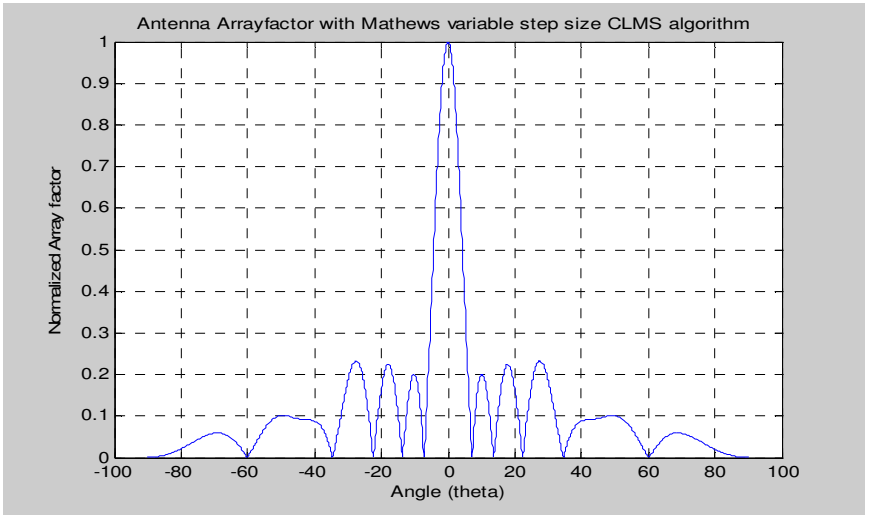

Figure 3. Antenna Array factor with Mathews variable step size CLMS algorithm

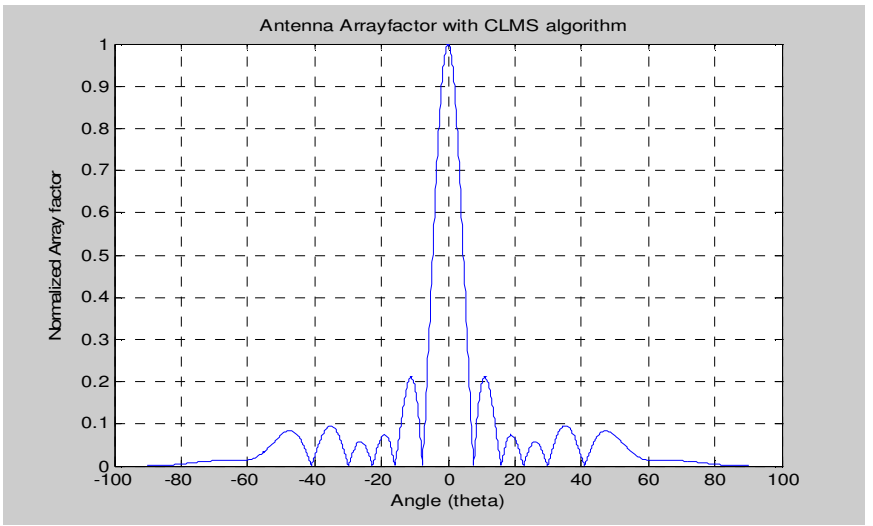

Figure 4. Antenna Array factor with CLMS algorithm

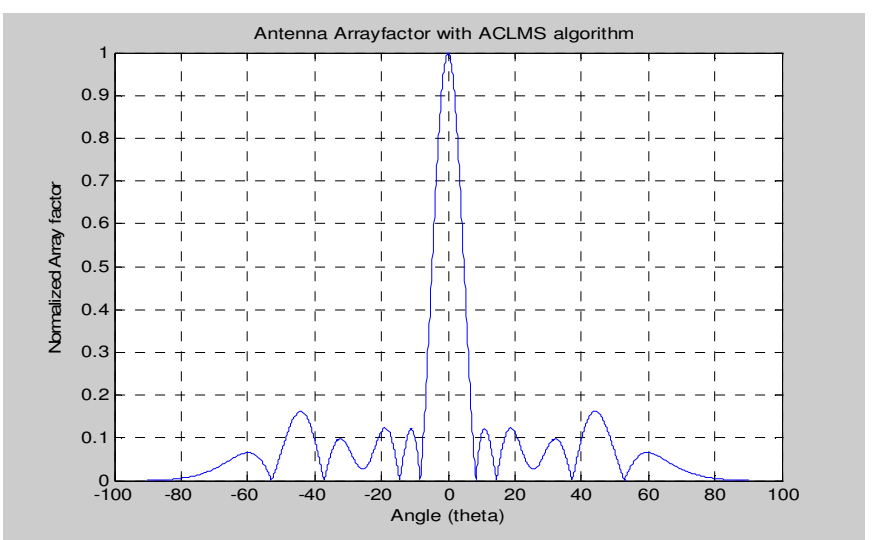

Figure 5. Antenna Array factor with ACLMS algorithm 


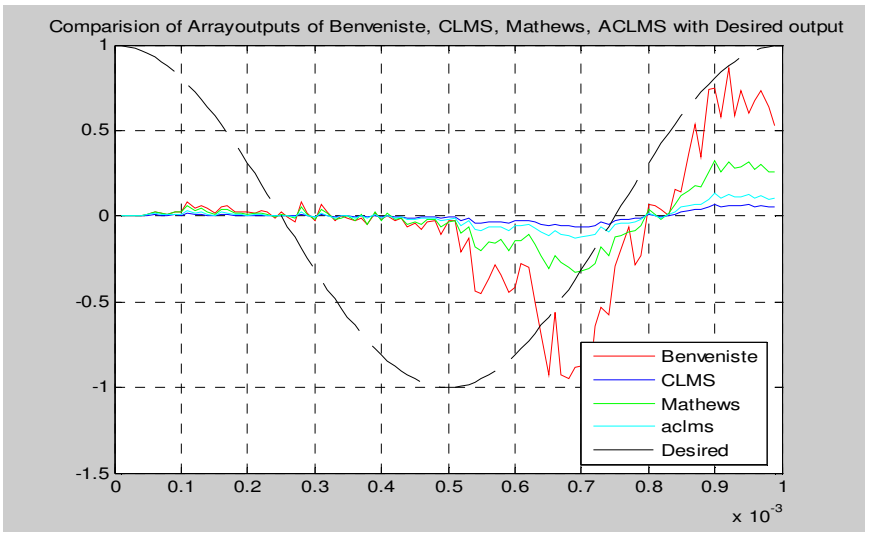

Figure 6. Signal convergence comparison in noisy environment

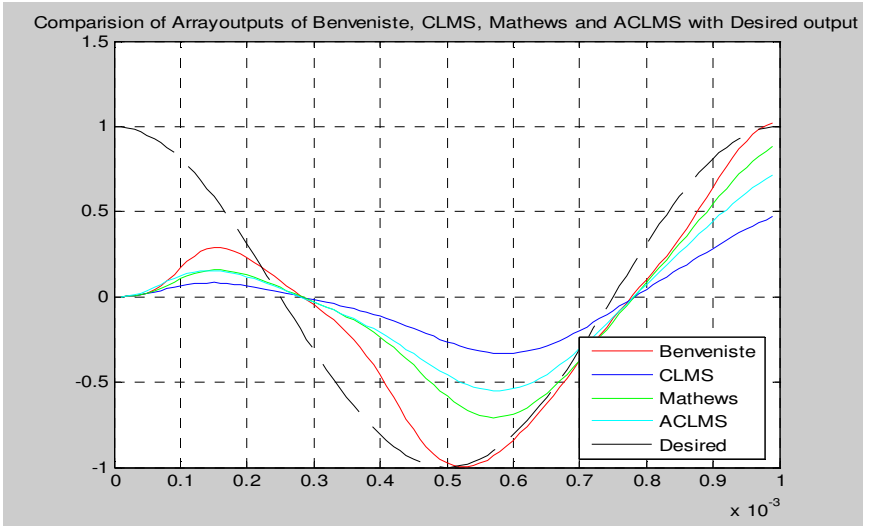

Figure 7. Signal convergence comparison in noiseless environment

\section{CONCLUSiON}

In noisy environment, the ACLMS gives better performance than CLMS with small stepsize parameter [7]. By using the variable adaptive stepsize algorithms with Benveniste and Mathews models as heuristics, a new approach has been designed for fast convergence and also resulting in low HPBW and SLL for smart antenna applications in the noisy environment. Out of these two heuristics, Benveniste model may be opted along with CLMS or ACLMS for better performance of Smart Antenna.

\section{REFERENCES}

[1] L.C. Godara, "Applications of Antenna Arrays to Mobile Communications. I. Performance Improvement, Feasibility and Considerations", IEEE Proceedings, Vol. 85, No.7, pp. 1031-1060, 1997.

[2] A. J. Paulraj, D. Gesbert, C. Papadias, "Smart Antennas for Mobile Communications", Encyclopedia for Electrical Engineering, John Wiley Publishing Co., pp. 1-15, 2000.

[3] Andrew I. Hanna and D. P. Mandic, "A complex-valued nonlinear neural adaptive filter with a gradient adaptive amplitude of the activation function", Journal of Neural Networks, vol. 16, issue 2, pp. 155-159, 2003.

[4] Su Lee Goh, and Danilo P. Mandic, "A Class of Gradient-Adaptive Step-Size Algorithms for Complex-Valued Nonlinear Neural Adaptive Filters", IEEE International Conference on Acoustics, Speech, and Signal Processing (ICASSP 2005), Philadelphia, 2005, pp. 253-256. 
[5] Danilo P. Mandic, Andrew I. Hanna, and Moe Razaz, “A Normalized Gradient Descent Algorithm for Nonlinear Adaptive Filters Using a Gradient Adaptive Step Size”, IEEE Transactions on Signal Processing, Vol. 8, No. 11, November 2001, pp 295-297.

[6] V. John Mathews, "A Stochastic Gradient Adaptive Filter with Gradient Adaptive Step-Size", IEEE Transactions on Signal Processing, Vol. 41, No. 6, June 1993, pp 2075-2087.

[7] Y. Ramakrishna, PESN Krishna Prasad, P.V. Subbaiah and B. Prabhakara Rao, "A Performance Analysis of CLMS and Augmented CLMS Algorithms for Smart Antennas ", Proc. 4th International Workshop on Computer Networks and Communications, Coimbatore 2012, pp. 9-19, DOI: 10.5121/csit.2012.2402.

[8] Wee-peng Ang and B. Farang-Boroujeny, "A New Class of Gradient Adaptive Step-Size LMS Algorithms", IEEE Transactions on Signal Processing, Vol. 49, No. 4, April 2001, pp. 805-810.

[9] Y. Ramakrishna, P.V. Subbaiah, B. Prabhakara Rao and PESN Krishna Prasad "Adaptive Nonlinear Gradient Decent (ANGD) Algorithm for Smart Antenna", International Journal of Mobile Network Communications and Telematics, Vol. 2, No. 6, December 2012, pp. 11-19, DOI: 10.5121/ijmnct.2012.2602.

\section{Authors}

Y. Ramakrishna received the B.E. degree and the M.Tech. degree in Electronics and Communication engineering from the University of Madras and Acarya Nagarjuna University in 2002 and 2005, respectively. He is currently pursuing the Ph.D. degree at JNT University Kakinada. He is now a Associate Professor in department of ECE, Prasad V. Potluri Siddhartha Institute of Technology, Vijayawada, India His research interests include interest includes Smart Antennas, Antennas and Wave Propagation, Mobile Communications and Microwave Engineering.

Dr. P. V. Subbaiah received his Ph.D. in Microwave Antennas from JNTU, India, 1995. His Master's Degree in Control Systems from Andhra University, India, 1982. He is Currently Working as Principal at Amrita Sai Institute of Science and Technology, Vijayawada, India since 2007. His Research interest includes Microwave Antennas, Optical Communications and Mobile Communications.

Dr. B. Prabhakara Rao received his Ph.D. from IISc Bangalore, India. He is currently working as professor in the department of Electronics and Communication Engineering. He has more than 30 years of experience in teaching and 24 years of R \& D. He is an expert in signal processing \& Communications. He produced $7 \mathrm{PhD}$ 's and guiding $20 \mathrm{Phd}$ scholars. He held different positions in his career like head of the department, vice principal, in JNTU College of engineering, Director (Institute of Science \& Technology),

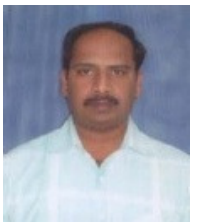
Director of Evaluation and Director (Foreign Universities and Alumni relations) in JNT University from 2003. He published more than 120 technical papers in national and international journals and conferences. His areas of interests are Wireless Communications, Digital Communications, Image Processing, Optical Networks, Network Security etc. 